\title{
El liberacionismo en Oceanía: Una cartografía de circulación de ideas suramericanas en el Pacífico Sur*
}

\author{
The liberationist thinking in Oceania: Cartography of circulación of Latin American \\ ideas in South Pacific
}

\author{
Eduardo Devés ${ }^{* *}$
}

\begin{abstract}
Resumen: La investigación consiste en detectar la presencia desde los años 1970s hasta inicios del siglo XXI, de dos expresiones del pensamiento liberacionista suramericano (la pedagogía de Paulo Freire y la teología de la liberación) en Oceanía. Se indican los ecosistemas intelectuales de recepción y algunas de las formas de circulación y procesos de reelaboración. Ello se estudia en 4 espacios: las instituciones de formación superior (universidades y seminarios religiosos), los movimientos de liberación, la educación de adultos y las organizaciones de pueblos indígenas. Se estudian casos en Estados y territorios como Australia, Fiyi, Nueva Caledonia, Nueva Zelanda, Papúa NG, Timor Oriental y Vanuatu. Se concluye caracterizando las formas en que las ideas suramericanas fueron recepcionadas-reelaboradas y los canales a través de los cuales llegaron a la región y por los cuales circularon dentro de la misma.
\end{abstract}

Palabras clave: Circulación de las ideas, Liberacionismo, Pensamiento latinoamericano, Paulo Freire, Oceanía

\begin{abstract}
The research consists of detecting the presence from the 1970s until the beginning of the 21st century, of two expressions of South American liberationist thinking (Paulo Freire's pedagogy and theology of liberation) in Oceania. Intellectual reception ecosystems and processes of circulation and re-elaboration are indicated in 4 intellectual spaces: higher education institutions (universities and religious seminaries), liberation movements, adult literacy, and indigenous peoples' organizations. Cases are detected in states and territories such as Australia, Fiji, New Caledonia, New Zealand, Papua New Guinea, Timor East and Vanuatu. Finally, this research concludes with the characterization of the ways in which the South American ideas were received and re-elaborated and the channels through which they arrived in the region and through which they circulated within it.
\end{abstract}

Keywords: Circulation of ideas, Liberationist thinking, Latin American thought, Paulo Freire, Oceania

Recibido: 15 agosto 2018 Aceptado: 3 octubre 2018

\footnotetext{
* Artículo producto Fondecyt 1150375. Todos los textos, cuya referencia se encuentra en lenguas diferentes del español, han sido traducidos por el autor.

** Profesor de IDEA-USACH, Instituto de Estudios Avanzados de la Universidad de Santiago de Chile, eduardo.deves@usach.cl,usach.academia.edu/EduardoDevés
} 


\section{Introducción}

Los estudios acerca de las ideas apenas se han aventurado hacia el Pacífico, entendido como el conjunto de la cuenca y menos hacia el interior de la cuenca. Prácticamente, la región del Pacífico Sur no ha sido unidad de análisis, sin menoscabo de pocas excepciones (Vervoorn 2005, Gardner 2013). Por otra parte, en el pensamiento suramericano y en los estudios acerca de este, el tema del Pacífico es completamente irrelevante, si se le compara con la importancia del Atlántico. Los estudios sobre el pensamiento suramericano se han ocupado recientemente de estudiar conexiones con otras regiones del mundo además de las relaciones convencionales con Europa Occidental (Beigel 2013, Bayle 2015, Devés 2017). Sin embargo, la cobertura espacial y regional es todavía escasa. Dentro de esto, el Pacífico se encuentra casi del todo ausente, salvo pocas excepciones (Zea 1988, Devés 2016, Rothwell 2012, Connelly 1983). Una primera mirada, insinuaría que no ha existido prácticamente circulación de las ideas y ello conduciría a un error importante, pues nuevas aproximaciones sugieren numerosas pistas. Avanzar en la tarea estudiar las conexiones de idas y venidas entre las riberas de la cuenca parece una necesidad urgente, especialmente para quienes quieren destacar dimensiones no eurocéntricas en la circulación de las ideas. Los objetivos que animan esta investigación son, en primer lugar, y de modo predominante, determinar en qué medioambientes intelectuales, de qué carácter y en qué Estados o territorios se recepcionaron y cultivaron aspectos del pensamiento liberacionista suramericano ${ }^{1}$; en segundo lugar, y de modo secundario, determinar desde dónde llegaron tales ideas, a través de qué canales y si desde allí fueron exportadas y hacia dónde. En relación a tales objetivos se trata de ofrecer una primera cartografía de circulaciones-instalaciones de ideas procedentes de Suramérica entre 1970, es decir, desde que los liberacionistas (Paulo Freire y los teólogos) publicaron sus primeros volúmenes, hasta poco más acá del 2000 .

El artículo se contenta con entregar algunos casos, teniendo en cuenta los diferentes ámbitos de recepción. No podría ser exhaustivo por la extensión requerida, sino que pretende ser solamente indicativo de lo que ocurre con una tendencia eidética suramericana en la región, aportando criterios de trabajo más que una revisión acabada. Debe agregarse que se han privilegiado las informaciones más tempranas por considerarse más interesantes.

Se seguirá una exposición histórica teniendo en cuenta grosso modo el orden en que se detecta esta presencia. Cada caso se estudiará considerando los asuntos siguientes: personas, instituciones, ciudades, años más relevantes y conexiones con otras figuras e instituciones, para entender esta circulación eidética, dejando hablar a quienes han protagonizado la recepción y la circulación de las ideas.

La hipótesis sobre la base de la cual se organizó esta investigación fue la existencia de numerosos nichos intelectuales en Oceanía, donde se habían recepcionado algunas ideas de origen suramericano, proceso de circulación eidética energizado por el Consejo Mundial de Iglesias (CMI). Como conclusión de una investigación más exhaustiva, esta hipótesis en buena medida se confirma, aunque se revelan otros casos con perfiles diversos, otras redes y "energizadores", es decir, instancias que dispusieron de recursos humanos y económicos para que la circulación se realizara. A partir de aquí, se clasifican los casos relevados de acuerdo a los ecosistemas intelectuales que los reciben: ámbitos académicos, universitarios y de seminarios de formación religiosa, movimientos de liberación nacional, movimientos indígenas y educadores en la formación de adultos. Tales ámbitos y nichos deben considerarse de modo

\footnotetext{
${ }^{1}$ Entenderé por liberacionismo el conjunto de expresiones suramericanas inspiradas en la noción liberación que se han expresado en la educación, la teología, la filosofía, la psicología, la sociología y entre otras. Quienes se interesen en este pensamiento pueden ver Cerutti 2007, Silva 2009, McKeever 2004, Richard, 2002, Dussel 1999, Gadotti 1996, Libanio 1992.
} 
interconectado, tanto porque las personas pertenecen a unos y a otros como, sobre todo, porque las ideas circulan entre unos y otros ${ }^{2}$.

\section{1-Universidades y seminarios teológicos}

Un primer ámbito de recepción se advierte en los seminarios teológicos de diversas confesiones cristianas y en varias universidades oceánicas. Un caso muy temprano se revela a partir del vanuatuense John Sethy Regenvanu, de confesión presbiteriana. En su biografía Regenvanu, nacido en 1945 y que llegó a desempeñar importantes cargos en la política del país antes y luego de su independencia, cuenta que durante sus estudios en Suva, capital de Fiyi, compartió con profesores y estudiantes de diversas instituciones educativas, tales como la Universidad del Pacífico Sur, donde estudiaba un pequeño grupo de vanuatenses y la gente del Movimiento Cristiano Estudiantil. En los años 1970s vivió en Fiyi como estudiante en el Pacific Theological College ${ }^{3}$, donde tuvo oportunidad en 1972 de escuchar a Ivan Illich durante su visita Suva (ver Gardner 2013, 135). Escribe Regenvanu:

"A través de estos contactos e intercambios de ideas, llegué a interesarme crecientemente en los problemas que afectaban a nuestra sociedad y ansioso de involucrarme activamente en los cambios. Comencé a escuchar acerca del padre Walter Lini y su periódico, Puntos de vista de las Nuevas Hébridas, e incluso en una ocasión me encontré con él en Suva junto a Kalpokor Lasakau. Esos jóvenes me inspiraron y los vi como los lideres con los cuales podría trabajar por el bien de nuestro país" (2004, 87).

Regenvanu cuenta lo que ocurrió a su vuelta a Port Vila (capital de Vanuatu) en 1974 y cómo se insertó en los quehaceres del Secretariado de Educación de la iglesia presbiteriana donde trabajaban los misioneros William y Roxianne Coop. Destaca Regenvanu que trabajar con ellos fue particularmente importante en su desarrollo: "Me motivaron a leer ampliamente: editoriales producidos por colegas de las islas del Pacífico, libros acerca de los países del Tercer Mundo, como aquellos escritos de Julius Nyerere en Tanzania o la Teología de la Liberación en América Latina” (2004, 88)". Abundando sobre esto, recuerda Regenvanu que estaba impactado por la propuesta de

“autosuficiencia (self-reliance) del Presidente Julius Nyerere de Tanzania. Igualmente estaba impactado por los conceptos de educación para la liberación y teología de la liberación desarrollados por los católicos romanos de América Latina, especialmente el doctor Paulo Freire. Freire cuyo método y tipo de trabajo con las masas política y socialmente oprimidas de Brasil, tenía un particular significado para mí" (2004, 92-93).

Lo anterior nos va mostrando numerosas pistas que apuntan hacia el CMI, que sabemos contribuía a la motivación, organización y financiamiento de un conjunto de iniciativas en la región y cuyas actividades ecuménicas en las diversas confesiones cristianas se expresaban en el envío de agentes de difusión del cristianismo, profesores para instituciones, financiamiento de viajes, publicaciones y becas, entre otras (ver Gardner 2013). Por lo demás Paulo Freire en esos años trabajaba en la sede central del CMI en Berna. Ello se hace más patente con el hecho que el CMI auspició muy temprano el viaje de Freire a Oceanía, ocasión en la cual habría participado en la conferencia sobre "Education for Liberation and Community" en el Pacific Theological College de Suva (Gardner 2013, 135, Hassan 1991, 9), en

\footnotetext{
2 Sobre esta conceptualización y algunos de sus desarrollos, puede verse Devés y Kozel (2018).

3 “The Pacific Theological College is an ecumenical theological college located in Suva, Fiji. Established in 1965, it opened for training in 1966 and was originally designed as the only regional institution to offer degree-level education in theology, available primarily to students from Pacific Island churches". Tomado desde www.http://ptc.ac.fj/.
} 
actividades en Australia y en el Seminario Waigani en Papúa NG en 1974, lugares donde ya se conocía su Pedagogía del Oprimido, publicada en inglés en 1970.

El vanuatense John S. Regenvanu apenas se refiere a las redes que circulaba las ideas suramericanas, redificando instituciones y publicaciones en la región, sin embargo, una revista como el Melanesian Journal of Theology las expresa más cabalmente. Esta revista se editó en la ciudad de Goroka, en Papúa Nueva Guinea, en el marco de las actividades del Instituto Melanesio y de la Asociación Melanesia de Colegios Teológicos. El australiano John D’Arcy May fue el editor de esta revista durante su primera época. Asociado al CMI, D’Arcy May residió en Papúa NG entre 1983 y 1987, luego de haber obtenido en la Universidad de Münster un doctorado en Teología y en Frankfurt otro en Historia de las Religiones. En la presentación al primer número de la revista, señalaba que la Melanesian Association of Theological Schools ha decidido crear "una revista con el propósito específico de desarrollar la teología indígena en Melanesia" (1985, 2). Claro está, la revista "implica el uso del inglés, que es ambas cosas, barrera e intermediario: que obstaculiza debido a su extranjerismo, como facilita por su universalidad. Este dilema es encarado por todos los teólogos del Tercer Mundo" (1985 a, 3). Además, insistió en esta orientación: "apuntamos a publicar regularmente reseñas bibliográficas con relevancia especial para Melanesia, particularmente en temas teológicos del Tercer Mundo" (1985a, 4). Algunas de estas ideas eran reforzadas en una reseña escrita por el mismo D’Arcy May comentando el libro de Walbert Buehlmann, The Coming of the Third Church, afirma que "una cosa fascinante es que esos énfasis diferentes y aparentes contradicciones han sido trabajadas en una discusión Sur/Sur entre los teólogos de cada lado, por ejemplo, en el foro de la Asociación de Teólogos del Tercer Mundo (EATWOT sigla en inglés) y en su periódico Voices of the Third World. Podemos ver hacia adelante el día en que los melanesios puedan realizar sus propias contribuciones a estas discusiones" (1985 b, 67). En este marco cita reiteradamente a Hugo Assman, Juan Luis Segundo y a Leonardo Boff, además del esrilanqués Aloysius Pieris, quien publicó Teología Asiática de la Liberación y participó tempranamente de las reuniones de la EATWOT, y a quien conocía personalmente. Debe notarse que la EATWOT es la red principal que ha articulado el liberacionismo teológico suramericano con el mundo

Paralelamente, analizando los primeros números del Melanesian Journal of Theology es posible darse cuenta cómo para su director y numerosas personas entre las que publicaban, la perspectiva, los temas, los autores y las redes del liberacionismo suramericano eran recurrentes. Incluso más, que ya existía cierto procesamiento no suramericano del liberacionismo, como esto de ir considerando temas como las teologías del tercer mundo, las teologías del Sur y la cuestión del contextualismo, que no corresponden propiamente a la primera etapa del liberacionismo suramericano. Ello por otra parte, muestra redes a través de las cuales circulaban las ideas liberacionistas, en algunos casos como asunto de militancia en favor de estas ideas, otras veces como conocimiento del estado de la cuestión teológica. Por ejemplo, Paul Richardson, un británico residente en Popondetta, ciudad de Papúa, se refirió a Gustavo Gutiérrez, "quien reclama que la teología de la liberación ofrece una nueva vía para hacer teología. Esta teología es mucho más autoconsciente acerca de su relación con las luchas cotidianas que la teología occidental. Aunque incluso la teología europea puede ser vista como respuesta a las presiones de la sociedad" $(1985,62)$. El artículo del tanzano Laurenti Magesa, por su parte, menciona el tema de la teología de la liberación, a Gutiérrez a Leonardo Boff, en relación al asunto del pecado social y la lucha de clases (1985, 176-177). Por su parte Albert Bundervoet, belga de origen, obispo católico de Rabaul, en Papúa NG, sostiene que si la evangelización es efectiva "nunca debe prescindir del contexto social y cultural en el cual se ubica o de la situación vital de aquellos hacia los que va dirigida. Es por esto que la teología de la liberación, tal como ha sido elaborada en América Latina no es y no puede ser un artículo para la exportación" (1985, 183-184). Por otra parte, en la revista no falta el autor que se haga cargo de la trayectoria de figuras oceánicas que o habían conocido el liberacionismo suramericano o tenían posiciones cercanas como John Momis, Walter Lini, Fred Timakata y Leslie Boseto. Esau Tuza escribe: 
"A nivel social y religioso John Momis y Leslie Boseto nos han hablado con voz fuerte y clara. Momis, un religioso católico, considera dentro de su rol como sacerdote envolverse con cuestiones relativas a la justicia social. Para él, es en la arena política de Papúa NG que la liberación del pueblo debe ser considerada. La relación iglesia-estado ha sido siempre un tema candente de diálogo, para Momis, como también para el religioso Walter Lini (anglicano) y al reverendo Fred Timakata (presbiteriano) de Vanuatu, quienes lucharon para conducir sus pueblos hacia la Independencia en 1980” (1985, 52).

En esta línea de estudiar las expresiones del liberacionismo y sus redes en el medio académico de Oceanía otra figura que debe mencionarse es el teólogo Philip Gibbs, quien muestra también un conjunto de recepciones, redes y reelaboraciones del liberacionismo suramericano en esos ámbitos académicos. Nacido en Nueva Zelanda en 1947, Gibbs obtuvo su doctorado en teología en la Universidad Gregoriana de Roma, con una tesis doctoral en torno a The Word in the Third World. Divine Revelation in the Theology of Jean-Marc Éla, Aloysius Pieris and Gustavo Gutiérrez. Gibbs ha dedicado numerosos trabajos a la teología de la liberación suramericana así como sus derivaciones hacia otros lugares del mundo y las eventuales modulaciones específicas que ha gozado allí (1998, 2005, 2007), ocupándose de las derivaciones del quehacer teológico desde Suramérica hacia Oceanía. En 1998 planteaba que "la teología de la liberación es un intento reciente tratando de integrar salvación y esfuerzo humano, pues trata la salvación como una realidad intra-histórica (donde) lo político es injertado en lo eterno" (1998, 42). Lo que Gibbs llamó “el debate feroz acerca de la teología de la liberación" es indicativo, "de la variedad de opiniones dentro de la iglesia Católica". Señalaba que

“en el pasado, las conservadoras iglesias Evangélicas y Pentecostales tendieron a considerar la política como un mundo pecaminoso y a mantenerse a distancia. Sin embargo, recientemente en Papúa NG ha habido un cambio significativo. Los cristianos han asumido que tienen que ser parte de las respuestas a sus oraciones. Ello significa que deben involucrarse en las realidades políticas y sociales" $(1998,42)$.

En esta línea citaba al decano Sherman quien escribía: "Estamos llamados a construir y a reparar el daño en los muros de la sociedad a través de la lucha, la oración y el involucramiento". Su llamado hacia el involucramiento no es diferente a la exigencia de "compromiso" en la teología de la liberación $(1998,42)$. En 2005 el neozelandés destacaba que "existen teologías contextuales viniendo desde muchos países del tercer mundo que pueden estimular ideas en el pueblo de Papúa NG. Los teólogos de Papúa NG deben hacer una contribución significativa al trabajo de la EATWOT" $(2005,51)$. Aludiendo a Aloysius Pieris, el inventor de la "teología asiática de la liberación", señalaba que hablando de teología contextual "es útil preguntarse acerca de cuanto encuentra la reflexión teológica en la experiencia de fe de las comunidades de base, lo que Pieris llama el tercer magisterio" (2005, 52). En 2007, Gibbs volvía sobre estos asuntos destacando que "en los últimos 30 años, ha habido numerosos intentos en varios lugares del mundo para incluir la circunstancia en la reflexión teológica", enfatizando que esta circunstancia es específica puesto que "en América Latina, la atención en la lucha social y política contra la dependencia y la pobreza ha resultado en una forma de teología en la cual la "liberación" ha llegado a ser la clave hermenéutica fundamental para entender el mensaje cristiano", en tanto que "en el contexto de Asia, el pluralismo religioso permanece como un constante desafío a la teología contextual" y, por su parte "en África, las teologías se han focalizado en las dimensiones étnicas y culturales de la vida en ese continente. En el Pacifico, ha habido renovada atención por desarrollar una genuina teología contextual” $(2007,4)$.

\section{2- La educación popular y de adultos}

Un segundo espacio de recepción-reelaboración del liberacionismo suramericano en Oceanía está constituido por quienes se ocupan de la educación popular y de adultos. La educación popular es un 
ámbito privilegiado y el más específico de recepción de las ideas de Paulo Freire. En relación a esto pueden relevarse varias expresiones y particularmente 3: una, la más convencional, que se entiende como labor pedagógica de educadores profesionales; otra, como educación indígena y que se traslapa con las organizaciones de pueblos originarios y; una tercera fórmula que se encuentra con los movimientos de liberación, como se constatará para Timor. En este acápite, solo se abordará la primera, pues las otras dos aparecen en los siguientes, con mayor propiedad.

La figura y las investigaciones en el marco de una tesis de doctorado de Gwayeweng Kiki, guiado por Edmund Parker en la Charles Sturt University de Australia, permiten ligar muy estrechamente la presencia del liberacionismo en seminarios y universidades con la educación de adultos. Kiki, de Papúa NG, se ha ocupado de la manera de enseñar teología a personas "no-occidentales". Durante una extensa residencia en el Pacific Theological College de Suva, realizó una investigación que caracterizó como orientada hacia "una mejor y más eficiente manera de enseñar teología a los estudiantes de Papúa NG dentro de la Iglesia Evangélica Luterana" (Parker y Kiki 2014, 108). Para formular y realizar sus investigaciones, acudió sistemáticamente a la obra de Paulo Freire como va dejando constancia en su trabajo ${ }^{4}$. Ha señalado que "de importancia particular fue un análisis de las metodologías de enseñanza y aprendizaje actuales usadas en la formación de los pastores luteranos en Papúa NG", que ello fue complementado con "la praxis y aprendizaje transcultural, que muestra maneras de conocer y aprender como son usadas por personas con orientaciones no occidentales. En esta línea fueron considerados escritores como Paulo Freire" y algunos otros. En tercer lugar, destacan la noción "wokabaut-karikulum, un 'concepto' proveniente del tokpisin (un pidgin de Papúa NG). En esta mirada, los teóricos no indígenas de educación, pedagogía y currículo fueron considerados junto a especialistas indígenas melanesios sobre cultura, religión y teología" (Parker y Kiki 2014, 109-110). Sus ideas, apuntando hacia sistemas epistemológicos melanesios, fueron innovadoras intentando articular el pensamiento de Freire con la trayectoria cultural melanesia, a partir del wokabaut-karikulum que se define como "un enfoque relacional compartido de aprendizaje que enfatiza la relación entre aprender y ser, en sentido que mente y cuerpo están comprometidos. Pero, por otra parte, el conocimiento compromete al individuo en su relación con la comunidad, donde la existencia del conocimiento, es socialmente distribuido" (Parker y Kiki 2014. 115).

La investigación acerca de la educación teológica de adultos recién destacada es muy reciente, pero el impacto de Paulo Freire en el ámbito de educación de adultos en Oceanía se advierte muy temprano, desde inicios de los 1970s. El suramericano visitó Melbourne en 1972, invitado como conferencista por el Consejo Mundial de Iglesias. Se ha sostenido (Osmond 2016) que su visita motivó el compromiso de los educadores populares del estado de Victoria con la educación de adultos como un quehacer de cambio social, implicando además que conceptos como "emancipación” y "liberación” permanecieran mucho tiempo en el discurso de este sector de educadores. Ello derivó en un conjunto de enfoques pedagógicos como: la co-participación en el aprendizaje, la concepción de la tarea del profesor como facilitador no como experto, la necesidad de tomar insumos de las vidas y experiencias de los estudiantes, la participación de estudiantes en la elaboración de objetivos y contenidos del aprendizaje, y la presencia de conceptos como emancipación y liberación en el discurso de quienes ejercían la profesión de educadores populares (Osmond 2016, Medlin 2017).

Precisamente como expresión de la larga herencia dejada en Australia por Freire, debe citarse la "Popular Education Network of Australia” y el grupo "Twenty First Century Critical Education” compuesto por Tracey Ollis, Jo Williams, Rob Townsend, Anne Harris, Jorge Jorquera y Lea Campbell, quienes en su declaración de principios destacaban que "inspirándose en las filosofías y escritos de Paulo Freire respecto

4 Por ejemplo: "Paulo Freire, Cultural Action for Freedom, preface by Joao da Veiga Coutinho (Middlesex: Penguin Education, 1974), and Pedagogy of the Oppressed, new rev. 20th-ann. ed. Trad por Myra Bergman (Ramos, New York: Continuum, 1997)”. 
a educación y activismo, la red, fundada en 2009, comprende a educadores, académicos y trabajadores comunitarios que trabajan en asuntos relativos a pedagogía crítica y cambio social en escuelas, comunidades y espacios de educación de adultos". Luego destacaban que el primer simposio que organizaron, acera de, Enseñanza y Aprendizaje para la Justicia Social y la Acción, "fue celebrado en la Universidad de Victoria en octubre 2010, orientado hacia las vías por las cuales la educación popular apoya una agenda de justicia social y funciones sociales dentro del desarrollo comunitario" y que allí la charla fundamental del simposio "fue impartida por la profesora Antonia Darder acerca de Reinventing Paulo Freire: A Critical Pedagogy of Love" (Ollis et al, 2010).

En esta misma línea y continuando todavía la herencia de Freire, debe citarse también el caso de la reunión del "Australian Council of Adult Literacy" realizada en Tasmania en 2012. Allí se planteó el tema de la campaña inspirada explícitamente en las ideas de Paulo Freire, teniendo en cuenta experiencias anteriores como las de Cuba, Tanzania, Nicaragua, Guinea Bissau, India y Timor Oriental, de la cual se ocupa específicamente el acápite siguiente. En el informe de la campaña en Tasmania, presentado por Bob Boughton (2012), se alude sistemáticamente a la obra de Freire en diversas regiones del mundo tanto como a autores que hicieron referencia al educador brasileño.

\section{3-Movimientos de liberación}

Un tercer ámbito de recepción lo constituyen los movimientos de liberación o independencia, particularmente en Timor Oriental y en Nueva Caledonia, como también, menos explícitamente, en Vanuatu. Estos fueron espacios donde las ideas del liberacionismo suramericano fueron acogidas e incluso reelaboradas en conexión con otras, existentes en dichos ecosistemas intelectuales, contribuyendo a inspirar tales movimientos. Por cierto, en diversos lugares, los tipos de lucha por la independencia fueron también diversos, además de exitosos o fracasados, y también lo fueron las ideas del liberacionismo que llegaron a esos lugares. En algunos casos se trató principalmente del pensamiento educacional freiriano, en otros este se asoció al pensamiento teológico.

En Timor Leste, el CMI, por lo general tan influyente en la circulación de las ideas liberacionistas, y los agentes de las principales iglesias actuantes en la región no jugaron papeles destacados. La circulación de las ideas liberacionistas implicó aquí otras redes. Un modelo muy distinto de circulación eidética se produce a través de estudiantes timorenses matriculados en algunas universidades de Portugal y comprometidos con la independencia, de lo que luego sería la República Democrática de Timor Oriental. En este caso, las organizaciones independentistas y la guerrilla asumieron los planteamientos de Paulo Freire como métodos para promover la alfabetización y la politización de las masas, ello a partir de la "Casa dos Timores", la organización estudiantil que los reunía en Lisboa. Allí se imprimió el manual de alfabetización "Timor es nuestro país", elaborado por el Frente Timorense de Liberación Nacional (FRETILIN) uniendo el afán pedagógico con el independentista. En este documento trabajaron, entre otros, Antonio Carvarinho y Francisco Borja da Costa (ver Leach 2017, 63-64). Ahora bien, "para que fuese posible toda una campaña de alfabetización, Silva (2012, p. 2) destaca que el FRETILIN, en asociación con el Comité Portugués de Descolonización, estableció el "Grupo de Coordenaçao para Reformulaçao do Ensino em Timor” (ver Urban 2017, 86). Según Silva (2011, p. 63), entre quienes integraban esta comisión para la alfabetización se encontraba la profesora portuguesa Judith Magalhaes quien, juntamente con los estudiantes de la "Casa dos Timores", de Lisboa, fue una de las personas que llevó las ideas de Paulo Freire sobre concientización en 1974 hacia la Unión Nacional de Estudiantes de los Timores (Silva, 2012, 2, Urban 2017, 86). Samuel Urban $(2017,87)$ destaca que fue precisamente "a través de este comité portugués que llegó a Timor la obra Pedagogía del Oprimido de Paulo Freire, siendo la base para la campaña de alfabetización" y que, más adelante, "los integrantes de la Casa dos Timores participarían del FRETILIN, siendo estos también 'formadores' (en la campaña de alfabetización), junto 
a gente de otras agrupaciones como los propios miembros de la Unión de Estudiantes Timorenses y algunas personas del Comité Portugués de Descolonización".

Diferente fue el caso del movimiento de independencia o autonomía en Nueva Caledonia, donde se trató más bien del liberacionismo teológico, en el marco de una red articulada a Fiyi, Vanuatu y Papúa y al CMI, más que a la fórmula timorense, aunque la similitud se advierte por la búsqueda de independencia y por la recepción del pensamiento suramericano en grupos de estudiantes realizando estadías en el extranjero. El caledonio Pothin Wete elaboró su obra más importante para este efecto, como tesis para sus estudios de postgrado en el Pacific Theological College en Suva, como había ocurrido más de una década antes con J. Regenvanu. Allí en 1987 presentó su trabajo sobre la posibilidad de una teología kanak de la liberación ${ }^{5}$. Charles W. Forman $(2005,118)$ afirma que Wete pasaba revista a la base histórica de la situación política, económica y social presente del pueblo kanak y la iglesia evangélica, trazando la emergencia de las instancias eclesiales en favor de la independencia, haciendo referencia a las teologías de la liberación de otras partes del mundo, apuntando hacia una teología kanak de la liberación. Según Forman, Wete llevó “el tema de la liberación a un nivel más fundamental”, en la medida que planteaba que la teología kanak de la liberación enriquecería y profundizaría las teologías de la liberación de otras tierras". Ello pues "esta expresaba el grito de un pueblo no solo por su liberación política y económica, sino por la existencia misma”. Según Wete, los kanaks estarían "en riesgo de perder su existencia a través de la pérdida de sus valores tradicionales y la adopción de costumbres foráneas". Y esta inquietud se afirmaba "no simplemente en la identidad nacional, sino que en raíces bíblicas. La demanda por independencia fluye de todo lo que los profetas hebreos han dicho acerca de justicia y libertad. Está relacionada también con la esperanza cristiana en una nueva creación. Esta esperanza conduce a los kanaks por el camino hacia la independencia”.

Wete continuó (Ver Forman 2005) una línea que ya antes había sido cultivada en la misma institución por otro kanak, Djoubelly Wea, quien se ocupó de una educación para la liberación del pueblo kanak ${ }^{6}$. Luego de sus estudios, Wea sobre todo, aunque también Wete, se implicaron en los movimientos independentistas e identitarios en Nueva Caledonia. Pothin Wete fue luego profesor del Pacific Theological College, cosa que muestra la presencia allí por décadas de una línea de pensamiento claramente afín al liberacionismo suramericano. En varios otros lugares del Pacífico Sur, figuras importantes de los movimientos independentistas, varias de estas pertenecientes al clero de diversas confesiones, como en el caso de Nueva Caledonia, tuvieron posiciones muy próximas y específicamente inspiradas en parte, en el liberacionismo, como se ha visto más arriba: John Momis, Walter Lini, Fred Timakata y Leslie Boseto.

\section{4-Las organizaciones de pueblos originarios}

El cuarto espacio está constituido por las organizaciones de pueblos originarios. En este ámbito, el proceso de toma de conciencia de la diferencia respecto de la etnia caucásica, predominante en Nueva Zelanda y en Australia, ha pasado entre otras cosas por las nociones de Paulo Freire. En este quehacer destaca la figura de Graham Hingangaroa-Smith, dirigente de los movimientos maoríes y profesor de la Universidad de Auckland. Su aproximación más importante al asunto ha sido la educación maorí y la coordinación de este pueblo con otros originarios en torno al Pacífico, además de los oceánicos, en lugares como Canadá, Hawái, USA continental, Taiwán y Chile, a través de lo cual se ha ligado al proyecto de las "Tribal Universities". Hingangaroa-Smith (2003) ha argumentado que la "contra-estrategia a la

5 Wete, Pothin. The development of the political awareness of the Kanak. Evangelical Church in New Caledonia and the Loyalty Islands from 1960 to 1987 and its theological implications possibility for a Kanak. liberation theology, Thesis (B.D.), Pacific Theological College, 1988.

${ }^{6}$ Publicada en 1977: An Education for the Kanak Liberation: A Project, Pacific Theological College 
hegemonía consiste en que los pueblos indígenas necesitan concientizarse críticamente a sí mismos, acerca de sus necesidades, aspiraciones y preferencias". Justifica "este llamado a una liberación (freeingup) de la imaginación y el pensamiento indígena" en razón que "uno de los elementos de la colonización consiste en la disminución de la habilidad indígena para imaginar la libertad o la visión utópica libre de opresión. De este modo un elemento crítico en la 'revolución' es la lucha por nuestras mentes, la liberación (freeing) de la mente indígena”. Quiere, sin embargo, articular el discurso proveniente de Paulo Freire con la trayectoria del pensamiento indígena, lo que denomina "enfoque Kaupapa Maor". Ello afirmado en que un término predominante como "descolonización" es una "noción reactiva", en tanto que "al ir hacia una política de transformación necesitamos entender la historia de la colonización, pero el grueso de nuestros trabajo y enfoque debe ubicarse en la imaginación de un futuro". Es ese sentido señala "la lección del enfoque Kaupapa Maori de Nueva Zelanda es que la transformación comprende dos partes: la confrontación con el colonizador y la confrontación con nosotros mismos, el modelo adentroafuera, que empalma con la noción de Freire de primero liberarnos nosotros mismos para liberar a otros" (2003). Hingangaroa-Smith ha continuado trabajando sobre el pensamiento-resistencia-praxis maorí sobre la base de Paulo Freire, como lo muestran sus publicaciones posteriores de 2009 y 2015 , pero sobre todo de 2017, en las cuales vuelve sobre temas relativos a Freire y la concientización. A HingangaroaSmith, pueden agregarse otras personas que trabajan en los mismos asuntos en Nueva Zelanda, como Tangiwai Kepa, quien presentó su tesis doctoral en la Universidad de Auckland en 2001, acerca de Language matters: The politics of teaching immigrant adolescents school English, donde da cuenta de la perspectiva con la cual ha enfrentado su investigación destacando que "esta tesis ha sido escrita desde la perspectiva de una profesora indígena maorí", que a pesar de haber sido "entrenada en los enfoques tecnocráticos de las prácticas", viene "buscando los aspectos de su cultura íntima, las formas de representarse el mundo de Tonga y Samoa y la pedagogía crítica de Paulo Freire, para transformar la educación contemporánea, que tiende a excluir el adolescente del aprendizaje en la escuela" (2001).

\section{Conclusiones}

Se ha ofrecido una primera cartografía de la presencia de ideas liberacionistas suramericanas en varios lugares de Oceanía. No son los únicos. Podría ampliarse la cantidad ecosistemas intelectuales y especificarse mejor los nichos a los cuales llegaron y en los que prosperaron estas ideas. Se trata de un primer recuento, realizado con un criterio pragmático y que permiten establecer algunas conclusiones que se pasa a detallar.

Acerca del tipo de los medioambientes intelectuales donde se recepcionaron y cultivaron. Se han determinado cuatro ámbitos principales (ámbito académico en universidades y seminarios, movimientos de liberación e independencia nacional, espacios de educación de adultos y organizaciones de pueblos originarios) en 7 estados o territorios del Pacífico Sur (Australia, Fiyi, Nueva Caledonia, Nueva Zelanda, Papúa NG, Timor Oriental y Vanuatu). Tales ámbitos no están completamente separados e interactúan e incluso se traslapan. Se ha optado únicamente por Oceanía buscando una homogeneidad en el tipo de ecosistemas intelectuales, aunque podrían haberse abordado otros casos del sur del Pacífico. Existen estudios que dan cuenta de la recepción del pensamiento de Paulo Freire, en particular en Filipinas (Cortez 2014 y 2013) y en Indonesia (Nuryatno 2006). Especialmente diferente habría sido el caso indonesio, pues se trata de la recepción de Freire en ecosistemas intelectuales que operan en lenguas autóctonas y marcados por el islam.

Acerca de los canales a través de los cuales llegaron las ideas suramericanas a diversos ecosistemas intelectuales oceánicos y si desde allí fueron exportadas o circuladas y hacia dónde, se concluye que, dado que se trata de ecosistemas intelectuales donde las lenguas de las intelectualidades son casi exclusivamente 
el inglés y el francés, es claro que la comunicación no siempre fue directamente desde Suramérica a Oceanía, ni siquiera en el caso de Timor Leste donde el idioma de la intelectualidad es el portugués. En todos los casos la circulación estuvo parcialmente mediada por redes y casas editoriales de Europa Occidental y USA, como puede verse por las traducciones, por la institucionalidad, por las redes. Ello por cierto, no excluye la agencia de figuras latinoamericanas que trabajaron en diversos lugares del Pacífico, especialmente en algunas ciudades de Australia y Nueva Zelanda, o que participaron en cargos relevantes en redes internacionales como es el caso del propio Paulo Freire, como también de José Miguez Bonino y Julio Santa-Ana (ver Paredes y Escalante 2010, Devés 2016) en el CMI, y sobre todo en la constitución y gestión de la EATWOT, clave en la circulación de las ideas liberacionistas entre diversos lugares del mundo periférico. Más específicamente, respondiendo a la cuestión de las redes que articularon a quienes recepcionaron y a los energizadores de la circulación, debe destacarse la diferencia entre teólogos de Australia y Nueva Zelanda, y quienes operaban en los ecosistemas de las islas. Los primeros tuvieron circulación en el primer mundo, conectados desde relativamente temprano a la EATWOT, y allí con gente de Suramérica y de otras procedencias donde se conocía el liberacionismo; respecto de los segundos, debe considerarse en primer lugar, la institucionalidad del Pacific Theological College en Suva, Fiyi, y la red que articuló a quienes enseñaron y/o estudiaron allí con otros ex-estudiantes y con numerosas personas participantes de diversas confesiones cristianas. El Pacific Theological College se revela como la institución probablemente más importante donde se recepcionaron, cultivaron y reexportaron las ideas del liberacionismo suramericano en la región del Pacífico Sur. Se ha visto como convergieron allí estudiantes y docentes de diversas procedencias y cómo, al menos en algunos casos, estas ideas fueron exportadas a Nueva Caledonia, por ejemplo. Ahora bien, si históricamente los energizadores, que han sostenido conscientemente o no la circulación de las ideas han sido agentes muy diversos: estados o imperios, diplomacia, instituciones educacionales, misioneros, propagandistas, debe señalarse que en este caso se ha tratado principalmente de iglesias y de otros agentes de la sociedad civil, sin menoscabo que los recursos económicos en muchas ocasiones dependieran de los estados y los financiamientos que estos otorgan a iglesias y ONGs. En todo caso, no debe imaginarse solo grandes energizadores que posibilitaron la circulación sino también numerosos casos de personas ligadas a la educación popular, a las organizaciones indígenas, a la política, a la docencia universitaria, a las prácticas eclesiales, entre otras actividades, que entusiasmadas por estas ideas decidieron transformarse en difusoras o "aplicadoras" como es el caso de la educación de adultos en Australia o Timor Leste.

\section{Bibliografía}

Apple, Michael (1999) "Freire, neo-liberalism and education" en revista Discourse: studies in the culturalpolitics of education vol 20, n 1

Bayle, Paola (2015) Conectando sures. La construcción de redes académicas entre América Latina y África, DOI: http://dx.doi.org/10.17141/iconos.53.2015.1445

Beigel, Fernanda (2013) "Centros y periferias en la circulación internacional del conocimiento" en Nueva Sociedad, n 245, Caracas

Boughton, Bob (2012) "South-South Cooperation: Can it work in Australia? Keynote Presentation Australian Council of Adult Literacy Annual Conference Hobart, Tasmania. 19-21 September 2012", Universidad de New England

Bundervoet, Albert (1985) "Liberation Theology in the Context of Papua New Guinea" Journal of Melanesian Association of Theological Schools, Vol 1, No 2, Goroka 
Cerutti, Horacio (2007) La filosofía de la liberación, FCE, México

Connelly, Marisela (1983) "Influencia del pensamiento de Mao en América Latina" Estudios de Asia y África Vol. 18, No. 2 (56), abril-junio

Cortez, Franz Giuseppe F. (2014) “The Prospect of Liberating Pedagogy in the Thoughts of Amable G. Tuibeo" Mabini Review, Polytechnic University of the Philippines, Vol 3, No. 1

Cortez, Franz Giuseppe F. (2013) “The Philippine Engagement with Paulo Freire” KRITIKE, Vol 7, No 2, diciembre 50-70 en http://www.kritike.org/journal/issue_13/cortez_december2013.pdf

Devés, Eduardo y Kozel Andrés (2018) Estudios Eidéticos. Una conversación, desde el sur, sobre la vida de las ideas y la reconfiguración de un espacio disciplinar, Ariadna, Santiago-Chile

Devés, Eduardo (2017) “La teología asiática de la liberación, un caso de recepción, apropiación y reelaboración de ideas" Universum, julio, vol.31, no.1

Devés, Eduardo (2016) "La circulación de las ideas, una conceptualización: el caso de la teología latinoamericana en Corea del Sur", Revista Estudios Avanzados, n 41, julio

Dussel, Enrique (1999) Teología da Libertação. Um panorama de seu desenvolvimento, Vozes, Petrópolis

Forman, Charles W. (2005) 'Finding Our Own Voice: The Reinterpreting of Christianity by Oceanian Theologians" International Bulletin of Missionary Research, Vol. 29, n 3, julio

Gadotti, Moacir (1996) Paulo Freire uma biobibliografia, Cortez Editora, Sao Paulo

Gardner, Helen (2013) "Praying for Independence. The Presbyterian Church in the Decolonisation of Vanuatu" The Journal of Pacific History, vol. 48, n 2

Gibbs, Philip (1998): “The Religious Factor in Papua New Guinea Politics” Catalyst 28/1

Gibbs, Philip (2005) Melanesian Journal of Theology, n 21-1 (2005)

Gibbs, Philip (2007) Narrative and Context in a Practical Theology for Papua New Guinea" Australian eJournal of Theology, n 9, marzo

Hassan, Graham (1991) “Church and state in Vanuatu 1945-1980: A 'Pacific' contest for power", en South Pacific Journal of Mission Studies, diciembre

Kepa, Tangiwai (2001) Language matters: The politics of teaching immigrant adolescents school English, Tesis PhD, Universidad de Auckland

Leach, Michael (2017) Nation-Building and National Identity in Timor-Leste, Routledge, Londres y Nueva York

Libanio João Batista (1992) "Panorama da teología da América Latina nos últimos 20 anos", Perspectiva Teológica 24, No 63, mayo-agosto 
Magesa, Laurenti (1985) "Instruction on the "Theology of Liberation": A Comment" Journal of Melanesian Association of Theological Schools, Vol 1, No 2, Goroka

May, John D’Arcy (1985, a) “Editorial: Introducing MJT”, en Journal of Melanesian Association of Theological Schools, Vol 1, No 1, Goroka

May, John D’Arcy $(1985$, b) THEOLOGIES OF THE “THIRD CHURCH” en Journal of Melanesian Association of Theological Schools, Vol 1, No 1, Goroka

McKeever, Martin (2004) “Thirty years of liberation theology”, Theology Digest $\mathrm{n} 51$

Medlin, Joanne (2017) “The Australian literacy and numeracy workforce: a literature review”, Occasional paper, NCVER, Adelaide

Nuryatno, Muhammad Agus (2006) Education and Social Transformation: Investigating the Influence and Reception of Paulo Freire in Indonesia $\mathrm{Ph}$. D. Thesis Faculty of Education, McGill University

Cortez, Franz Giuseppe F. (2014) "The Prospect of Liberating Pedagogy in the Thoughts of Amable G. Tuibeo" Mabini Review, Polytechnic University of the Philippines, Vol 3, No. 1, 16-69

Ollis, Tracey, Jo Williams, Rob Townsend, Anne Harris, Jorge Jorquera y Lea Campbell (2010) "The Popular Education Network of Australia (PENA) and Twenty-First-Century Critical Education" en Peters, Michael y Tina Besley Paulo Freire. The global legacy, Peter Lang Publishing Books, Berlin

Osmond, Pamela (2016) "What happened to our community of practice? The early development of adult basic education in NSW through the lens of professional practice theory" en Literacy and Numeracy Studies, vol 24, n 2

Paredes, Alejandro y Eduardo Escalante (2010) La visualización de “colegios invisibles" en las publicaciones político-religiosas de editorial Tierra Nueva (década 1970) y su inserción en discursos de época" en revista Theoria, Vol. 19 (1), Universidad del Bio-Bio

Parker, Ermund y Kiki, Gwayaweng (2014) "Is There a Better Way to Teach Theology to Non-Western Persons? Research from Papua New Guinea that Could Benefit the Wider Pacific" Australian eJournal of Theology 21.2

Regenvanu, John (2004) From Village to Nation: an Autobiography, Institute of Pacific Studies and Emaulus Campus. U. of the South Pacific, Suva

https://books.google.cl/books?id=0WEeYDxeE9gC\&printsec=frontcover\&hl=es\&source=gbs_ge_s ummary_r\&cad $=0 \# \mathrm{v}=$ onepage $\& \mathrm{q} \& \mathrm{f}=$ false

Richard, Pablo (2002) "La Iglesia y la Teología de la Liberación en América Latina y el Caribe: 19622002”, Pasos 103, setiembre-octubre

Richardson, Paul (1985) Seeing Western Theology in Context, Journal of Melanesian Association of Theological Schools, Vol 1, No 2, Goroka

Rothwell, Matthew (2012) Transpacific Revolutionaries: The Chinese Revolution in Latin America, Routledge

Schugurensky, Daniel (2014) Paulo Freire, Bloomsbury, Londres 
Silva, Antero Benedito da (2012) "Literacy Model of the Maubere Pedagogy", Comunicação apresentada no Grupo de Estudos Brasil-Timor, Peace and Conflict Studies Institute

Silva, Antero Benedito da (2011) FRETILIN Popular Education 1973-1978 and its relevance to Timor-Leste today, Tesis Doctorado, Universidad New England

Silva, Sergio (2009) La Teología de la Liberación” en revista Teología y Vida, Vol. L

Smith G. Hingangaroa (2003) "Indigenous Struggle for the Transformation of Education and Schooling, Keynote Address to the Alaskan Federation of Natives Convention". Anchorage, Alaska

Smith G. Hingangaroa (2009) "Mai i te maramatanga, ki te putanga mai o te tahuritanga: From Conscientisation to transformation", en Andrzejewski, J., et al (Ed). Social Justice, peace and environmental education: transformative standards, Routledge, Nueva York y Londres

Smith G. Hingangaroa (2015) "Equity as Critical Praxis: The Self-Development of Te Whare Wananga o Awanuiarangi”" en Peters, M y T. Besley, Paulo Freire: The Global Legacy (Eds.) Peter Lang, New York

Smith G. Hingangaroa 2017 "Keynote address: 12 noon, Wednesday, July 26, University Theatre (ADM026) Academic Work as Transforming Praxis: From Discourse to Enactment (Show me the blisters on your hands!)" en http://rmooc.ca/news/july-26-keynote-130-pm-graham-hingangaroasmith/

Tuza, Esau (1985) "Instances of 'God-talks' in Melanesia” en Journal of Melanesian Association of Theological Schools, Vol 1, No 1, Goroka

Urban, Samuel (2017) "Paulo Freire e a educação popular em Timor-Leste: uma história de libertação" en Revista Educação e Emancipação, São Luís, v. 10, n. 1, enero/abril

Vervoorn, Aat (2005) “Intelectuales públicos en Asia y el Pacífico” Anuario Asia Pacifico, Barcelona

Wete, Pothin (1988) The development of the political awareness of the Kanak Evangelical Church in New Caledonia and the Loyalty Islands from 1960 to 1987 and its theological implications possibility for a Kanak liberation theology Thesis (B.D.), Pacific Theological College

Zea, Leopoldo (1988) "Prólogo" a Rizal, José Noli me Tangere, Biblioteca Ayacucho, Caracas 